\title{
Admission Control Methods in IMS Networks
}

\author{
Erik Chromy, Matej Kavacky and Lubomir Dresto
}

\begin{abstract}
In this paper we present admission control methods for IMS network. The task of RACS block is to accept or reject new connection into the network. The main goal of the admission control method is to ensure the Quality of Service not only for new connection but also for already accepted connections. We discuss and compare three admission control algorithms in the paper from the qualitative parameters point of view.
\end{abstract}

Keywords-Admission Control, MBAC, IMS, Quality of Service.

\section{IP Multimedia Subsystem}

IMS (IP Multimedia Subsystem) is a standardized NGN (Next Generation Network) architecture for the telecom operators in order to offer mobile and multimedia services such as IPTV (Internet Protocol Television), VoIP (Voice over Internet Protocol) and many others. The main goal of IMS is the provision of new services as well as all present and future services that can provide telecommunications network. Telecom operators can provide their services to users regardless of their location, access technology and terminals. To achieve these objectives, the IMS defines a complete architecture that enables the convergence of voice, video and data across the infrastructure based on IP protocol. This system allows interoperability between mobile and IP architecture, therefore the IMS system is independent of the access technology that supports packet-switched network such as GPRS (General Packet Radio Service), UMTS (Universal Mobile Telecommunications System), CDMA2000 (Code Division Multiple Access 2000), WLAN (Wireless Local Area Network), WiMAX (World Interoperability For Microwave Access) and DSL (Digital Subscriber Line). The older technologies such as PSTN (Public Switched Telephone Network) and GSM (Global System for Mobile Communications) are supported through the gateways. The main characteristic of the IMS architecture is the distribution of network infrastructure to the separate functions with standardized interfaces.

An important control function of NGN plays the control layer whose main goal is traffic routing, traffic acceptance or

Manuscript received July 22, 2016, revised August 30.

This article was created with the support of the Ministry of Education, Science, Research and Sport of the Slovak Republic within the KEGA agency project - 007STU-4/2016 named: Progressive educational methods in the field of telecommunications multiservice networks.

E. Chromy is with the Slovak University of Technology, Faculty of Electrical Engineering and Information Technology, Departement of Telecommunications, Ilkovicova 3, 81219 Bratislava, Slovakia (e-mail: chromy@ut.fei.stuba.sk).

M. Kavacky is with the Slovak University of Technology, Faculty of Electrical Engineering and Information Technology, Departement of Telecommunications, Ilkovicova 3, 81219 Bratislava, Slovakia (e-mail: kavacky@ut.fei.stuba.sk).

L. Dresto is with the Slovak University of Technology, Faculty of Electrical Engineering and Information Technology, Departement of Telecommunications, Ilkovicova 3, 81219 Bratislava, Slovakia (e-mail: lubdresto@gmail.com). billing information provision. The control layer also controls the user traffic transmitted through the RACS (Resource and Admission Control Sub-system) component [1]-[4].

\section{A. Resource and Admission Control Sub-system}

RACS is the most important logical network element which is intended to reserve resources, access control and support for QoS (Quality of Service). RACS block is composed of PDF (Policy Decision Function) and A-RACF (AccessResource and Admission Control Function) components. PDF implement local rules on the use of resources, it is used for example for overload protection of specific access media element and A-RACF controls the QoS network parameters.

\section{Admission Control Methods}

For the support of the real-time and multimedia applications, the QoS must be guaranteed in the network. The result of admission control is to accept or reject an incoming connection. The main criteria of admission control is to provide the QoS for new connection while maintaining QoS of already accepted connections [5]-[7].

$\mathrm{AC}$ methods can be divided into two categories: PBAC (Parameter Based Admission Control) and MBAC (Measurement Based Admission Control).

PBAC methods are applicable on total traffic characteristics such as for example peak transmission rate. Based on this focus the method determines the required network resources for all connections. MBAC methods focus on measurements of the current network traffic. This method accepts connections on the basis of measurements performed in the network [8].

The traffic characteristics of many applications are not known. In the case of small knowledge of traffic parameters, often the overstated bandwidth is allocated in order to ensure the required QoS. This leads to under utilization of network resources. Therefore the use of MBAC methods can ensure the better utilization of network resources and effective provision of the QoS.

The admission control is needed to accept new connections, respectively services. This control ensures QoS for the transmitted data. By use of admission control methods, it is possible to design a model of admission control that will ensure a certain quality of service. Created models may be used alone, or may be complementary (to achieve better QoS). The admission control of incoming flows is the most important step in order to ensure QoS. This control is provided by AC (Admission Control) methods. A key function of the AC methods is to estimate the anticipated bandwidth of incoming connection and the analysis of the current utilization of the network load, whereby it is possible to allocate a given bandwidth for connection. AC methods are mostly used for 
services sensitive to delay and jitter, respectively for real-time applications.

Various admission control methods have been proposed and their difference lies mainly in various types of functionality and method of implementation. Some AC methods are based on mathematical calculations and statistical indexes and the other on traffic measurements. To ensure required QoS the AC methods should fulfill certain conditions:

- they should avoid delay by fast decision process,

- they should not affect already accepted connections and ensure QoS of requesting connection,

- they should have simple implementation into the system,

- they should effective allocate required bandwidth to data flows and efficient utilization of the link capacity.

- they should effective allocate required bandwidth to data flows and efficient utilization of the link capacity.

The use of AC methods plays a key role particularly in the field of access networks, which unlike the core networks do not have such high transmission bandwidth. The actual information about calculated bandwidth is sent to border nodes, what produces unnecessary traffic in the core network. Therefore the AC methods are applied in the access part of the network [9].

\section{A. MBAC Methods}

The MBAC methods are based on measurements of current state of output network interface or actual network state in order to make decision if the new data flow should be accepted to the network. The MBAC methods accept new data flows until measurement results reach defined values of capacity (usually recommended value is $90 \%$ of network capacity). The measurement process is needed for use of MBAC methods [10]-[12].

For measurement realization we need to know the link utilization and only minimal knowledge of connection source. Decision process of MBAC methods is based on measurement of traffic and QoS parameters. Interval measurements ensure efficient resource utilization in the case of data bursts creation or when the data transmission is slower than the peak transmission rate.

\section{MBAC ALgORITHMS}

Various algorithms for admission control based on measurements have been proposed in the literature. Our paper addresses following algorithms:

- Simple Sum,

- Estimated Sum,

- Acceptance Region.

\section{A. Simple Sum}

Simple Sum is simple algorithm which ensures sufficient bandwidth for accepted flows. AC method accepts new data flow only in the case the following condition is true:

$$
v+r_{\alpha}<C
$$

where $v[\mathrm{kbit} / \mathrm{s}]$ denotes the sum of reserved transmission rates, $C$ [kbit/s] denotes link capacity, $\alpha$ is index for requesting data flow and $r_{\alpha}[\mathrm{kbit} / \mathrm{s}]$ denotes peak transmission rate of new data flow.

\section{B. Estimated Sum}

This algorithm samples traffic data during the defined intervals. These input samples represent the input data for prediction. After every sampling the prediction of future data transmission is done. Such prediction will be used for future connection acceptance or rejection. The decision process is based on the equation:

$$
\hat{y}(n+1)+p_{\alpha} \leq \mu \cdot C
$$

where, $\alpha$ denotes index for requesting data flow, $\hat{y}(n+1)$ [kbit/s] denotes prediction of aggregated traffic used in next sampling $(n+1), p_{\alpha}[\mathrm{kbit} / \mathrm{s}]$ denotes peak transmission rate of new flow, $C[\mathrm{kbit} / \mathrm{s}]$ is link capacity, $\mu$ represents the channel utilization.

\section{Acceptance Region}

This algorithm [10] predicts region of maximal link utilization at the expense of packet losses. Estimation of Acceptance region can be based on the parameters such as bandwidth, switch buffer, and parameters of stack buffer filters. The calculation of acceptance region assumes Poisson distribution of incoming independent requests. The algorithm ensures that the sum of measured traffic and transmission rates of new flows will not exceed the acceptance region.

$$
C(S)=\frac{1}{S} \cdot \log \left[1+\frac{v}{p}\left(e^{S p}-1\right)\right]
$$

where, $C[\mathrm{kbit} / \mathrm{s}]$ represents estimation of bandwidth for total traffic, $v[\mathrm{kbit} / \mathrm{s}]$ denotes average transmission rate of traffic, $p[\mathrm{kbit} / \mathrm{s}]$ denotes peak transmission rate, $S$ represents space parameter within the range $(0,1)$.

\section{Simulations}

The simulations of MBAC algorithms were performed in MATLAB environment. The principle of admission control methods is depicted in Fig. 1] where $n$ represents number of data flows (Variable Bit Ratel) and the maximal link capacity is set to $1 \mathrm{Mbit} / \mathrm{s}$ (sufficient for the simulation results). The AC method accepts only such number of flows which ensures that the maximal link bandwidth will be not exceeded.

Data flows come in random times. These data flows have different requirements on network in different times. It means that the peak transmission rate of particular data flows vary during time. In our paper we assume exponential distribution with mean value $(\lambda)$ set to $64[\mathrm{kbit} / \mathrm{s}]$. Current traffic is simulated with sample of 250 time points during which the loss parameter of particular flows is calculated. 


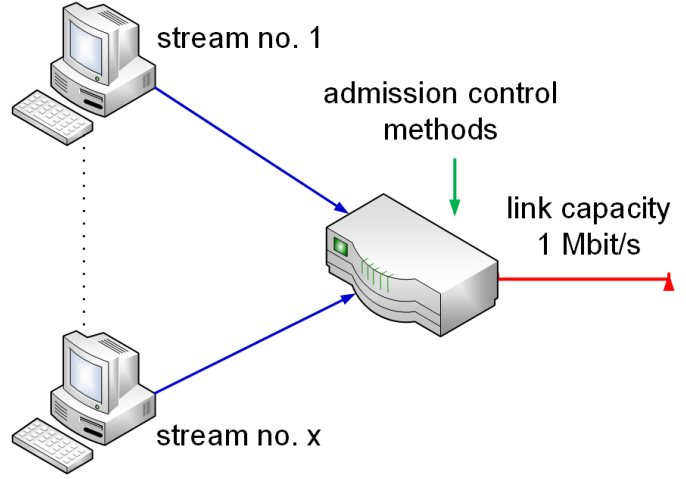

Fig. 1. Principle of admission control simulation

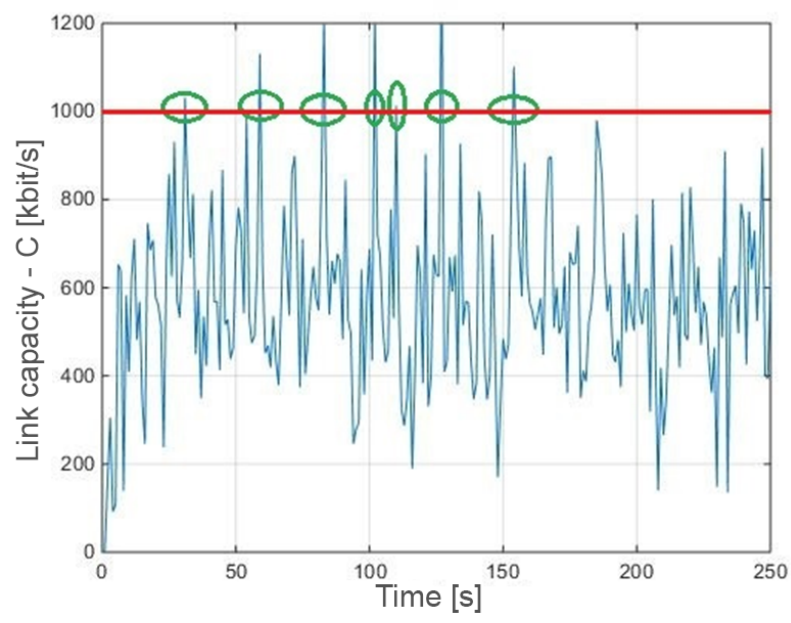

Fig. 2. Simulation results for Simple Sum algorithm

\section{A. Simple Sum Algorithm Simulation}

The Simple Sum is the simplest admission control algorithm. It accepts or rejects requesting connection according to available bandwidth. The simulation results for this algorithm are depicted in Fig. 2. The red line represents the maximal allowed link capacity and it is set to $1 \mathrm{Mbit} / \mathrm{s}$. This value can not be exceeded by decision result. The blue curve represents the current network traffic consisting of 10 accepted data flows (i.e. on the base of simulations, the Simple Sum algorithm has accepted 10 flows). Green circles are points in which link capacity was exceeded. When the link capacity is exceeded, packet losses will occur. The main task of AC method is to ensure QoS and it is not sufficiently ensured when there are packet losses. We can see in Fig. 2 that in some time points the link utilization is only around $50 \%$ of allowed capacity. The main factor of AC method evaluation in our paper is loss. The Simple Sum algorithm reached 3\% loss.

\section{B. Estimated Sum Algorithm Simulation}

Estimated Sum algorithm makes decision of acceptance or rejection of requesting connection based on estimation of the future traffic while uses only part of link capacity. In our simulation this part of link capacity was set to $90 \%$ of total link capacity (brown line in Fig. 3). The red line represents the maximal allowed link capacity. Blue curve represents the

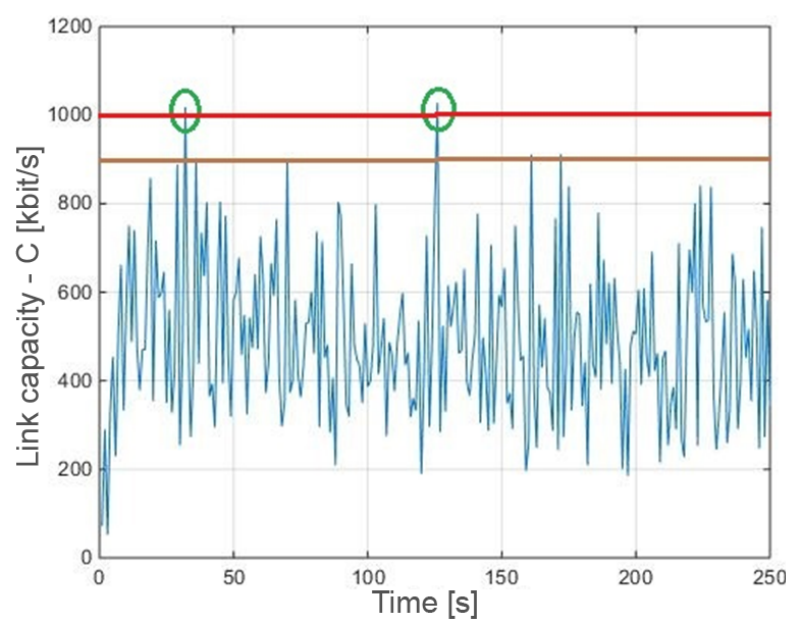

Fig. 3. Simulation results for Estimated Sum algorithm

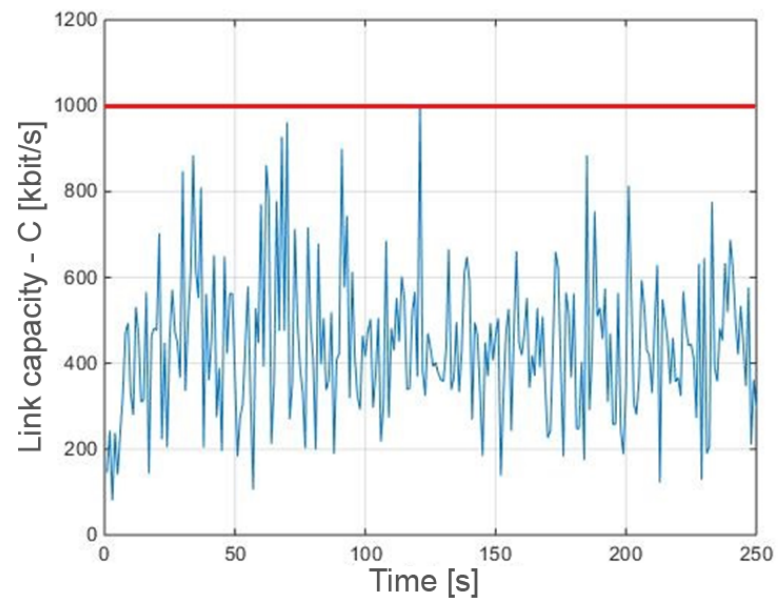

Fig. 4. Simulation results for Acceptance Region algorithm

current traffic consisting of 9 data flows accepted by Estimated Sum algorithm. Green circles are points in which available link capacity was exceeded. The Estimated Sum algorithm accepted 1 less connection than Simple sum algorithm, but the loss is only $1 \%$. From this point of view the Estimated Sum algorithm is better for admission control than Simple Sum algorithm.

\section{Acceptance Region Algorithm Simulation}

Simulation results for Acceptance Region algorithm are depicted in Fig. 4 Compared to Simple Sum and Estimated Sum algorithms this algorithm is more complicated because more input parameters are used. Decision process of this algorithm is based on area of maximal link utilization at the expense of data loss. Space parameter $S$ within the range $(0,1)$ is set to 0,5 due to most precise measurement. Red line represents the maximal allowed link capacity. Blue curve represents the current traffic consisting of 8 data flows accepted by this algorithm. It is the smallest number of accepted connections from performed simulations, but data loss is zero, therefore sufficient QoS is ensured. 


\section{Discusion}

Performed simulations demonstrate the field of admission control methods in IMS networks. Selected algorithms accept or reject connections into the network on the basis of different ways and methods. From the simulation results we can see that for given link capacity particular algorithms accept different number of connections while each of them aims to ensure some level of QoS. But not all of them can achieve the sufficient level of QoS. In our paper we have observed data loss as main parameter for comparison of simulated admission control algorithms.

The Simple Sum algorithm has accepted 10 data flows at the loss of $3 \%$. Estimated Sum algorithm has accepted 9 data flows at the loss of $1 \%$. The best algorithm from loss point of view appears the Acceptance Region with 8 accepted data flows and zero loss. Therefore only this last algorithm has achieved the required QoS in proposed network.

\section{CONCLUSION}

The admission control in IMS network is actual and perspective research subject today. The RACS block is network component in which admission control methods should be implemented. There is not any actual standard with recommendation which $\mathrm{AC}$ method should be preferred for the particular services.

In our paper we have observed three admission control algorithms. These algorithms are not used for IPTV or VoIP traffic. In our simulations we have observed these methods in general - the input flow with variable bit rate character was used. In the future work detailed analysis of selected AC methods for particular services will be performed.

\section{REFERENCES}

[1] ETSI Standard, ETSI ES 282003 V1.1.1(2006-06): Telecommunications and Internet converged Services and Protocols for Advanced Networking (TISPAN); Resource and Admission Control Sub-system (RACS); Functional Architecture

[2] H. Zeng, Y. Gao and Y. Xia, On NGN Architecture And Evolution Strategy, Innovations in NGN: Future Network and Services, 2008. KINGN 2008. First ITU-T Kaleidoscope Academic Conference, 2008, pp. 337-342, ISBN: 978-92-61-12441-0

[3] H. Allouch and M. Belkasmi, Design of distributed IMS by classification and evaluation of costs for secured architecture, Innovative Computing Technology (INTECH), 2012 Second International Conference on. 2012 , pp. 291-296, ISBN: 978-1-4673-2678-0

[4] M.A. Qadeer and A.H. Khan, IMS Network Architecture, International Conference on Future Computer and Communication, 2009, ICFCC 2009, pp. 329-333, ISBN: 978-0-7695-3591-3

[5] E. Chromy, M. Jadron, M. Kavacky and S. Klucik, Admission Control in IMS Networks, Advances in Multimedia, vol. 2013, Article ID 918930, 7 pages, 2013, ISSN:1687-5680 (Print),ISSN:1687-5699 (Online), DOI:10.1155/2013/918930

[6] A.W. Moore, Measurement- Based Management of Network Resources, A dissertation submitted for the degree of Doctor Philosophy of University Cambridge, June, 2001

[7] E. Chromy, M. Weber and T. Behul, Admission Control Methods and Quality of Service, Workshop of the 12th International Conference KTTO 2012, November, 2012, Malenovice, Czech Republic, pp. 36-40, ISBN 978-80-248-2810-7

[8] L. Yuan-Cheng and T. Sheng-Fu, Unfairness of Measurement-based Admission Controls in a Heterogeneous Environment, 8th International Conference on Parallel and Distributed Systems, ICPADS 2001, June, 2001, Kyongju City, pp. 667-674, ISBN: 0-7695-1153-8, DOI: 10.1109/ICPADS.2001.934882
[9] S. Jamin, S.J. Shenker and P.B. Danzig, Comparision of Measurementbased Admission Control Algorithms for Controlled-Load Service, INFOCOM '97, 16th Annual Joint Conference of the IEEE Computer and Communications Societies. Driving the Information Revolution, April, 1997, Kobe, pp. 973-980, ISBN: 0-8186-7780-5, DOI: 10.1109/INFCOM.1997.631035

[10] S. Jamin and S. Shenker, Measurement-based Admission Control Algorithms for Controlled-load Service: A Structural Examination, Michigan: University of Michigan, 1997, Technical Report.CSE-TR-333-97

[11] M. Egyhazy and Y. Liang, Predicted Sum: A Robust Measurement-Based Admission Control with Online Traffic Prediction, IEEE Communications Letters, 2007, Vol.11, Issue 2, pp.204-206, ISSN: 1089-7798, DOI 10.1109/LCOMM.2007.061127

[12] S.Y. Yerima, Implementation and Evaluation of Measurement-based Admission Control Schemes within a Converged networks QoS Management Framework, International Journal of Computer Networks \& Communications (IJCNC) Vol.3, No. 4, July, 2011, DOI: $10.5121 /$ ijcnc. 2011.3410

Erik Chromy was born in Velky Krtis, Slovakia, in 1981. He received the M.Sc. degree in telecommunications in 2005 from Faculty of Electrical Engineering and Information Technology of Slovak University of Technology (FEI STU) Bratislava. In 2007 he submitted PhD work. His scientific research is focused on queueing systems, contact center and admission control methods. Nowadays he works as assistant professor at the Institute of Telecommunications of FEI STU Bratislava.

Matej Kavacky was born in Nitra, Slovakia, in 1979. He received the M.Sc. degree in telecommunications in 2004 from Faculty of Electrical Engineering and Information Technology of Slovak University of Technology in Bratislava (FEI STU). In 2006 he submitted PhD. work Quality of Service in Broadband Networks. His scientific research is focused on admission control methods Nowadays he works as assistant professor at the Institute of Telecommunications of FEI STU Bratislava.

Lubomir Dresto was born in Ilava, Slovakia, in 1991. He received the M.Sc. degree in telecommunications in 2016 from FEEIT STU in Bratislava. His scientific research is focused on admission control methods. 